\title{
Traumatic brain injury is associated with increased syndecan-1 shedding in severely injured patients
}

Erika Gonzalez Rodriguez ${ }^{1 *}$ D, Jessica C. Cardenas ${ }^{1}$, Charles S. Cox ${ }^{4}$ Ryan S. Kitagawa ${ }^{5}$, Jakob Stensballe ${ }^{2,3}$, John B. Holcomb ${ }^{1}$, Pär I. Johansson ${ }^{1,2}$ and Charles E. Wade ${ }^{1}$

\begin{abstract}
Introduction: Head injury and exsanguination are the leading causes of death in trauma patients. Hemorrhagic shock triggers systemic endothelial glycocalyx breakdown, potentially leading to traumatic endotheliopathy (EOT). Levels of syndecan-1, a main glycocalyx component, have been used to assess the integrity of the glycocalyx. In TBI patients, it remains unclear whether syndecan-1 shedding occurs and its correlation with outcomes. We aimed to determine the frequency of EOT+, defined as a syndecan-1 level of $40 \mathrm{ng} / \mathrm{ml}$ or higher, after TBI in isolated and polytraumatic injury. We also investigated how the presence of EoT+ affected outcomes in TBI patients.
\end{abstract}

Methods: Severely injured trauma patients were enrolled. From blood samples collected upon patients' arrival to the hospital, we measured syndecan-1 (main biomarker of EoT+), soluble thrombomodulin (sTM, endothelial activation) adrenaline and noradrenaline (sympathoadrenal activation), and assessed TBI patients' coagulation capacity.

Results: Of the enrolled patients $(n=331)$, those with TBI and polytrauma $(n=68)$ had the highest rate of EoT+ compared to isolated TBI $(n=58)$ and Non-TBI patients $(n=205)$ (Polytrauma-TBI 55.9\% vs. Isolated-TBI 20.0\% vs. non-TBI polytrauma 40.0\%; $p=0.001$ ). TBI patients with EOT+ exhibited marked increases in STM, adrenaline and noradrenaline levels, and physiological and coagulation derangements. In isolated TBI patients, increasing syndecan1 levels ( $\beta$ for every $10 \mathrm{ng} / \mathrm{ml}$ increase: 0.14; 95\% Cl: 0.02, 0.26) and hypocoagulability were negatively associated with survival.

Conclusions: This study provides evidence of syndecan-1 shedding after TBI supporting the notion that breakdown of the glycocalyx contributes to the physiological derangements after TBI.

Keywords: Endothelium, Syndecan-1, Traumatic endotheliopathy, Sympathoadrenal activation

\section{Background}

Head injury and hemorrhagic shock are the leading causes of death in trauma patients [1]. Most of these deaths occur in the first $24 \mathrm{~h}$ following injury, and it is during this time window that interventions that could potentially improve outcomes should be implemented [2-4]. While the implementation of strategies such as a balanced 1:1:1 plasma: platelets: red blood cell ratio has

\footnotetext{
* Correspondence: Erika.r.gonzalez@uth.tmc.edu

${ }^{1}$ Center for Translational Injury Research (CeTIR), Department of Surgery,

McGovern Medical School, University of Texas Health Science Center, 6431

Fannin, MSB 5.204, Houston, TX 77030, USA

Full list of author information is available at the end of the article
}

considerably reduced early mortality caused by hemorrhage, the proportion of early deaths attributed to acute traumatic brain injury (TBI) has remained unchanged [1]. Early interventions are limited partly because of a lack of understanding of the systemic pathogenesis of both isolated TBI and TBI with polytrauma $[1,4,5]$.

Hemorrhagic shock leads to the systemic breakdown of the glycocalyx, a protective barrier on top of endothelial cells in blood vessels that is involved in the regulation of coagulation, inflammation, transcapillary flux, and mechanotransduction [6-8]. Alterations in these responses are believed to lead to traumatic endotheliopathy (EoT), a syndrome associated with high mortality [8-10]. Previous

(c) The Author(s). 2018 Open Access This article is distributed under the terms of the Creative Commons Attribution 4.0 International License (http://creativecommons.org/licenses/by/4.0/), which permits unrestricted use, distribution, and 
studies have demonstrated an association between the shedding of glycocalyx components and increased morbidity and mortality [11-13]. Using plasma samples collected from trauma patients, Rahbar et al. [11, 12] demonstrated that reductions in plasma oncotic pressure were associated with increased shedding of a major component of the glycocalyx, syndecan-1, and higher permeability (assessed in vitro). Patients with glycocalyx breakdown and lower plasma oncotic pressure were in shock, required a higher volume of blood transfusions, and had poorer survival than did patients without these characteristics [11, 12, 14]. Furthermore, a recent study from our laboratory demonstrated an association between acute low levels of serum albumin and increased glycocalyx breakdown in trauma patients [15]. The findings of these studies point to a state of increased permeability and endothelial dysfunction that leads to poorer outcomes [16-20].

The effects of shock and injury in the glycocalyx in patients with TBI are still not fully described, and whether the breakdown of the vascular glycocalyx can lead to EoT in TBI patients is unknown. In a porcine model of TBI and hemorrhage, Sillesen et al. [21] measured markers of endothelial activation and injury, inflammation, sympathoadrenal activation, and cell death. Syndecan-1 levels were significantly higher in animals subjected to TBI and hemorrhage than in controls. However, because the experimental group was exposed to both TBI and hemorrhage, whether the observed endotheliopathy resulted only from the systemic effects of hemorrhagic shock in the endothelium or also from the TBI-induced breakdown of the glycocalyx in cerebral blood vessels is unclear [21]. Further evidence suggested that the sympathoadrenal activation that occurs after head injury could drive the coagulopathic response seen in TBI patients [22, 23]. Di Battista and colleagues [23] also proposed a link between these responses and acutely increased levels of syndecan-1, as a biomarker of endotheliopathy, in isolated TBI patients with unfavorable outcomes.

Recently, we provided a quantitative definition of EoT and demonstrated that a cutoff syndecan-1 level of $40 \mathrm{ng} / \mathrm{ml}$ or higher $(\mathrm{EoT}+)$ identifies a group of patients who have an increased need for transfusion of blood products and higher mortality despite having similar admission physiology to patients without EoT [24]. However, the frequency of EoT, the factors associated with the development of this syndrome and its effects on outcomes in TBI patients are unknown. Here, we aimed to determine the frequency of EoT, defined as a syndecan-1 level of $40 \mathrm{ng} / \mathrm{ml}$ or higher, following TBI in the contexts of isolated and polytraumatic injury. We also investigated how the presence of glycocalyx breakdown, as measured by syndecan-1, affected outcomes in TBI patients. We hypothesized that head injury can lead to EoT and that the presence of severe TBI in combination with polytrauma potentiates glycocalyx breakdown as evidenced by syndecan-1 shedding. We expected that patients with both TBI and polytrauma would more frequently have EoT+ than would patients with either TBI alone or polytrauma alone. Identifying patients at risk of EoT paves the way for the development of clinical studies that investigate how best to treat patients in need of endothelial repair. Furthermore, this study contributes to the understanding of EoT and its effects on the clinical outcomes of TBI patients.

\section{Methods}

\section{Study design and sample analysis}

For this prospective observational study, we obtained approval from The University of Texas Health Science Center at Houston Institutional Review Board (HSC-GEN-12-0059). This study included previously reported data from 247 patients $[8,24,25]$. Adult patients who were admitted to our level 1 trauma center from July 2011 to May 2016 and who required trauma team activation were eligible for inclusion. Patients were excluded if they were pregnant, were prisoners, were enrolled in other studies, declined to consent, or if no blood sample was drawn on admission. Consent was obtained from the patient or a legally authorized representative within $72 \mathrm{~h}$ of admission or waived for patients who were discharged or died within $24 \mathrm{~h}$ of hospital admission. No changes in clinical practice were implemented in this observational study.

We collected $20 \mathrm{ml}$ of blood in citrated tubes upon patients' arrival to the emergency department. Blood was transferred into vacutainer tubes containing 3.2\% citrate and inverted to ensure proper anticoagulation. Rapid thrombelastography (rTEG) were performed according to the manufacturer's instructions. Samples were then centrifuged at $3200 \mathrm{rpm}$ for $20 \mathrm{~min}$ at room temperature. After spinning, plasma was aliquoted and frozen for later analysis. In addition, we collected blood samples from healthy female and male volunteers to serve as controls.

We only included patients with blunt trauma and an injury severity score (ISS) above 8 for whom syndecan-1 levels and other essential data were available. Syndecan-1 levels were also measured from 29 healthy volunteers. Of the 731 patients assessed for eligibility, 41 patients were excluded because of missing syndecan-1 level data, 96 patients were excluded because they had an ISS of less than 9, and 105 patients were excluded because they had penetrating trauma. Out of the excluded patients with penetrating injury, 11 had TBI. All of these patients sustained isolated injuries to the head $(82.0 \%$ gunshot wounds), and three subsequently died. Eight more patients in the TBI group were excluded because they were on anticoagulation at the time of injury (Fig. 1). The demographic profiles of the excluded patients with mild trauma $($ ISS $<9)$ were similar to those of patients included in the 


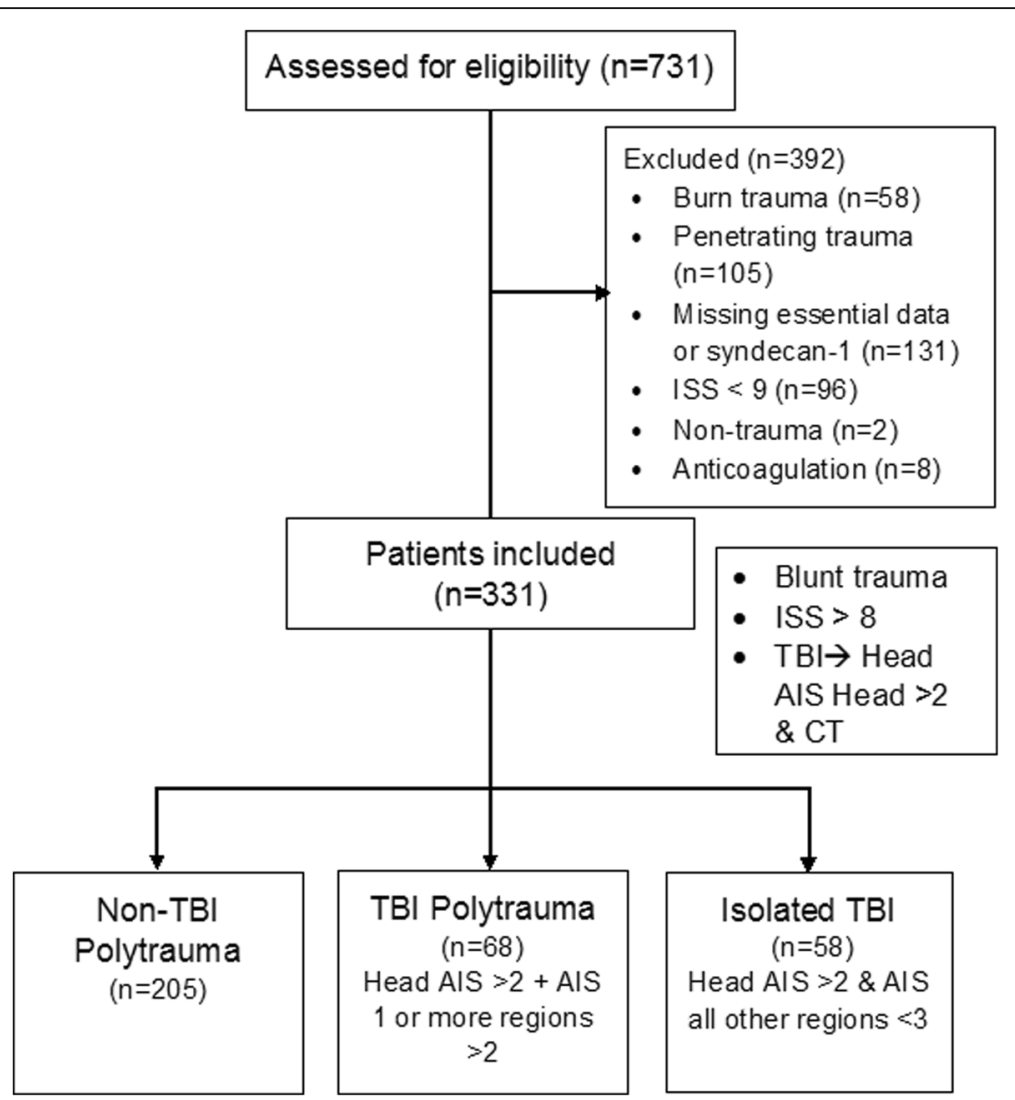

Fig. 1 Study flow diagram showing the selection process used to identify trauma patients for inclusion in the study. ISS, Injury Severity Score; TBI, traumatic brain injury; AIS, Abbreviated Injury Scale; CT, computed tomography

study and were representative of our trauma patient population (age: median 35 years, interquartile range [IQR] $27-53$ years; sex: $77 \%$ male; mortality rate: $10.4 \%)$. By comparing the study cohort to the overall trauma patient population, we ensured that we were not excluding any particular demographic group from the analysis, except for patients that suffered penetrating trauma. Furthermore, data from 240 patients included in this study have been previously reported by Johansson and colleagues $[8,25]$, and Gonzalez Rodriguez et al.

Patients were classified as having severe TBI if their Abbreviated Injury Scale (AIS) Head/Neck score was higher than 2, and there was radiological confirmation of TBI with computed tomography (CT). The attending trauma surgeon judges the need of obtaining an initial head CT scan based on arrival Glasgow Coma Score (GCS), mechanism of injury and clinical criteria. Subsequently, both a radiologist and the trauma team interpret the head CTs and request consultations from neurosurgery if needed. Patients with an AIS Head/Neck score higher than 2 and an AIS score in all other regions lower than 3 were classified as having isolated TBI. Patients with TBI and an AIS score in at least one region of more than 2 were classified as polytrauma-TBI. Patients without TBI, but with an AIS score in at least two regions higher than 2 were defined as non-TBI polytrauma. The types of brain lesion were assessed and classified into $5 \mathrm{mu}-$ tually exclusive categories: subarachnoid hemorrhage, subdural hemorrhage, epidural hematoma, intraparenchymal contusion, and multifocal intracranial hemorrhage $(\mathrm{MIH}$; in cases where 2 or more brain lesions were observed, but neither was dominant).

Designated study personnel prospectively collected patient demographics, vital signs, standard laboratory values, transfusions data, arrival GCS, and information on the mechanisms and severity of injuries at the time of admission. Outcomes, including hospital and intensive care unit-free days and 30-day in-hospital mortality, were obtained from medical records.

\section{Enzyme-linked immunosorbent assays}

Our main biomarker of interest, syndecan-1, was quantified from plasma obtained at hospital arrival using a commercially available enzyme-linked immunosorbent assay (Diaclone SAS, Besancon, France; lower limit detection $4.94 \mathrm{ng} / \mathrm{ml}$ ). We also measured: soluble thrombomodulin, a marker of endothelial cell activation or dysfunction, was quantified using a commercially available enzyme-linked 
immunosorbent assay (sTM Nordic Biosite, Copenhagen, Denmark; lower limit detection $0.31 \mathrm{ng} / \mathrm{ml}$ ) according to manufacturer recommendations. Adrenaline (2-CAT ELISA, Labor Diagnostica Nord GmbH \& Co. KG, Nordhorn, Germany; lower limit of detection $10 \mathrm{pg} / \mathrm{ml}$; normal reference level $<100 \rho g / \mathrm{ml}$ ) and noradrenaline (2-CAT ELISA, Labor Diagnostica Nord GmbH \& Co. KG, Nordhorn, Germany; lower limit of detection $50 \mathrm{pg} / \mathrm{ml}$; normal reference level $<600 \mathrm{pg} / \mathrm{ml}$ ) as indicators of sympathoadrenal activation and drivers of EoT $[8,25]$.

\section{Coagulation assessment by viscoelastic hemostatic assays} Rapid thrombelastography tests (rTEGs) were run on a Thrombelastograph 5000 (Hemoscope Corp, Niles, IL) by technicians at the Memorial Hermann Hospital emergency department, according to manufacturer's recommendations. The activated clotting time (ACT) was defined as the time from the start of the test to fibrin formation. Values equal to or more than $128 \mathrm{~s}$ are highly predictive of the need for massive transfusion within $6 \mathrm{~h}$ [26]. The maximum amplitude (MA) is indicative of the contribution of platelet function and platelet-fibrin interactions to clot strength (reference value: $54-72 \mathrm{~mm}$ ). LY30 is the percent amplitude reduction at $30 \mathrm{~min}$ after MA (reference value $0.0-7.5 \%$ ).

\section{Statistical analysis}

Sample size calculation was performed to detect a difference of syndecan-1 levels of at least $12 \mathrm{ng} / \mathrm{ml}$ between healthy volunteers and trauma patients' mean levels. We determined that at least 27 patients per group were needed. Based on our previous work, we used a cutoff syndecan-1 level of $\geq 40 \mathrm{ng} / \mathrm{ml}$ to define EoT+ [area under the curve $0.70,95 \%$ CI $0.58-0.84$ ) [24]. Mann-Whitney U or Kruskal-Wallis tests were used for comparisons between and across groups. Post-hoc analyses were performed with Dunn's tests. The chi-square test was used for comparisons between categorical variables. Summary statistics (medians with interquartile ranges (IQR)) were used to describe continuous variables, and categorical data were presented as frequencies and percentages. With multivariable logistic regression, we determined factors associated with the likelihood of 30-day mortality in patients with isolated TBI. A purposeful selection of covariates approach was followed to fit the model. Initially, we considered variables known to be clinically significant in trauma and TBI that were collected within $3 \mathrm{~h}$ of hospital arrival (demographic characteristics, vital signs, arrival physiology, and mechanism of injury). From the univariate analysis, we selected for inclusion in the preliminary effects model those variables that were significant at the $p<0.2$ level. Checks for interactions were made, and goodness of fit of the model was assessed with the Hosmer-Lemeshow goodness-of-fit test. Statistical significance was set at the $p<0.05$ level. All statistical analyses were performed with Stata software version 13.0 (StataCorp, College Station, TX).

\section{Results \\ Patient characteristics}

Of 581 patients with plasma samples available, 331 met the inclusion criteria. Forty percent of them $(n=126)$ had TBI (polytrauma, $n=68$; isolated TBI, $n=58$ ). Most patients in the complete cohort were moderately injured (ISS: median 22, IQR 16-29) males (71.3\%). These patients typically had altered mental status on arrival (GCS: median 5, IQR 3-15) but tended to be normotensive (systolic blood pressure: median $119 \mathrm{mmHg}$, IQR 99-140) and to have normal arrival hemoglobin levels (median 13.0, IQR 11.6-14.2 g/dl) and platelet counts (median 220, IQR 183-270). The rate of EoT+ (syndecan-1 level $\geq 40 \mathrm{ng} / \mathrm{ml}$ ) was $39.8 \%$, similar to the rate in our previous report [24]. Survival at 30 days was $79.5 \%$ (Table 1 ). In terms of type of brain lesion, $37.3 \%(n=47)$ of patients presented with subdural hemorrhage, 23.0\% $(n=29)$ with subarachnoid hemorrhage, $21.4 \%(n=27)$ with $\mathrm{MIH}, 7.1 \%(n=9)$ with epidural hematoma and $11.1 \%(n=14)$ with intraparenchymal hemorrhage.

\section{Syndecan-1 shedding in patients with isolated TBI,} polytrauma in combination with $\mathrm{TBl}$, and polytrauma only In order to determine whether TBI in the presence of polytrauma exacerbated the breakdown of the glycocalyx and resulted in a higher frequency of EoT+, we assessed and compared levels of our biomarker of interest (syndecan-1) across patients with isolated TBI, polytrauma and TBI, and polytrauma without TBI. Syndecan-1 levels in controls were significantly lower than in non-TBI polytrauma (median: $19.1 \mathrm{ng} / \mathrm{ml}$ vs. $31.5 \mathrm{ng} / \mathrm{ml} ; p=0.009$ ), isolated TBI (median: $19.1 \mathrm{ng} / \mathrm{ml}$ vs. $25.0 \mathrm{ng} / \mathrm{ml} ; p<0.05$ ) and polytrauma-TBI patients (median: $19.1 \mathrm{ng} / \mathrm{ml}$ vs. $54.0 \mathrm{ng} / \mathrm{ml} ; p=0.0001)$. We observed significant increases in shedding of syndecan-1 in polytrauma-TBI patients compared to those with polytrauma but no TBI (non-TBI polytrauma) $(p=0.001)$ and those with isolated TBI $(p<0.001)$. Median syndecan-1 levels in the polytraumaTBI group were 1.5 times higher than in the non-TBI polytrauma group $(54.0 \mathrm{ng} / \mathrm{ml}$ vs. $31.5 \mathrm{ng} / \mathrm{ml})$ and more than 2 times higher than those in the isolated TBI group (54.0 $\mathrm{ng} / \mathrm{ml}$ vs. $25.2 \mathrm{ng} / \mathrm{ml}$ ) (Fig. 2). Conversely, sTM levels were comparable across groups, indicating similar levels of endothelial activation or dysfunction. The majority of polytrauma-TBI patients were EoT $+(n=38$, $55.9 \%)$; in the other two groups, this proportion was significantly lower $(p=0.001)$ (Table 1$)$. 
Table 1 Demographic and clinical characteristics of 331 trauma patients

\begin{tabular}{|c|c|c|c|c|c|}
\hline & All patients $N=331$ & Non-TBI Polytrauma $N=205$ & Poly TBI N=68 & Isolated TB| $N=58$ & $P$ value \\
\hline \multicolumn{6}{|l|}{ Demography } \\
\hline Age, y & $43[29-58]^{a}$ & $42[27-57]$ & $44[34-58]$ & $50[33-76]$ & 0.12 \\
\hline Male, $n(\%)$ & $236(71.3)$ & $145(70.7)$ & $52(76.5)$ & $39(67.2)$ & 0.5 \\
\hline ISS & 22 [16-29] & 19 [14-27] & $34[27-41]^{d}$ & $25[16-26]^{e}$ & $<0.0001$ \\
\hline Head AIS = 3, n (\%) & $45(35.7)^{b}$ & - & $30(44.1)$ & $15(25.9)$ & 0.015 \\
\hline Head AIS = 4, n (\%) & $35(27.8)^{b}$ & - & $21(30.9)$ & $14(24.1)$ & \\
\hline Head AIS $\geq 5, n(\%)$ & $45(35.7)^{b}$ & - & $16(23.5)$ & $29(50.0)$ & \\
\hline \multicolumn{6}{|c|}{ ED Vital Signs and Laboratory Values } \\
\hline $\mathrm{SBP}, \mathrm{mmHg}$ & 119 [99-140] & $114[93-137]^{f}$ & 116 [100-139] & $140[128-158]^{e}$ & 0.0001 \\
\hline $\mathrm{HR}$, beats/min & $95[79-112]$ & $96[83-119]^{f}$ & $93[77-111]$ & $86[70-102]$ & 0.004 \\
\hline $\mathrm{BE}, \mathrm{mEq} / \mathrm{l}$ & $-4[-7$ to 0$]$ & $-4[-7 \text { to }-1]^{\mathrm{f}}$ & $-5[-8$ to -2$]$ & $-1[-5 \text { to } 2]^{e}$ & 0.0001 \\
\hline Platelet count, $10^{9} / \mathrm{I}$ & $221[185-270]$ & $224[189-278]^{f}$ & 222 [189-264] & 206 [172-256] & 0.2 \\
\hline Hemoglobin, g/dl & 13.0 [11.6-14.2] & $13.0[11.6-14.4]$ & 13.2 [11.5-13.9] & 13 [11.9-14.4] & 0.86 \\
\hline GCS & 6 [3-15] & $10[3-15]^{f}$ & $3[3-9]^{d}$ & 3 [3-9] & 0.0001 \\
\hline \multicolumn{6}{|l|}{ Biomarkers } \\
\hline sTM, ng/ml & $6.2[4.6-8.4]$ & $6.1[4.4-8.2]$ & $6.5[5.1-8.8]$ & $5.6[4.3-9.3]$ & 0.24 \\
\hline Syndecan-1, ng/ml & $33.2[16.6-79.1]$ & $31.5[15.5-61.2]$ & $54.0[25.1-107.0]^{d}$ & $25.0[15.5-44.4]^{\mathrm{e}}$ & 0.0007 \\
\hline EoT,$+ n(\%)$ & $132(39.8)$ & $82(40.0)$ & $38(55.9)^{d}$ & $12(20.6)^{e}$ & 0.001 \\
\hline \multicolumn{6}{|l|}{ Outcomes } \\
\hline Transfused, $n$ (\%) & $191(58.1)$ & $114(55.6)$ & $50(73.5)^{d}$ & $27(46.5)^{\mathrm{e}}$ & 0.014 \\
\hline 24-h mortality, n (\%) & $18(5.4)$ & $7(3.4)^{f}$ & $4(5.9)$ & $6(10.3)$ & 0.025 \\
\hline 48-h mortality, n (\%) & $37(11.2)$ & $16(7.8)^{f}$ & $9(13.2)$ & $12(20.7)$ & 0.02 \\
\hline 72-h mortality, $n(\%)$ & $40(12.1)$ & $16(7.8)^{f}$ & $11(16.2)^{d}$ & $13(22.4)$ & $<0.0001$ \\
\hline 30-day mortality, n (\%) & $68(20.5)$ & $27(13.2)^{f}$ & $19(27.9)^{d}$ & $22(37.9)$ & $<0.0001$ \\
\hline
\end{tabular}

Abbreviations: TBI traumatic brain injury, ISS Injury Severity Score, AIS Abbreviated Injury Score, ED emergency room, SBP systolic blood pressure, $H R$ heart rate, $B E$ base excess, GCS Glasgow Coma Scale, STM soluble thrombomodulin, EOT traumatic endotheliopathy

${ }^{a}$ Values are medians and interquartile ranges (IQR) unless otherwise specified

bonly in 126 patients

'Data for only 226 patients

${ }^{\mathrm{d} S t a t i s t i c a l l y ~ s i g n i f i c a n t ~ d i f f e r e n c e ~ b e t w e e n ~ t h e ~ P o l y t r a u m a ~ T B I ~ a n d ~ N o n-T B I ~ p o l y t r a u m a ~ g r o u p s ~}$

eStatistically significant difference between the Polytrauma TBI and Isolated TBI groups

fStatistically significant difference between the Non-TBI and Isolated TBI groups

\section{Subgroup comparisons between EoT+ and EoT-patients with TBI}

We stratified TBI patients according to the presence $($ EoT +$)$ or absence (EoT-) of EoT. EoT + patients $(n=50,39.7 \%)$ had higher ISS, base deficit, and were more likely to be tachycardic, hypotensive, and coagulopathic on arrival than were EoT- patients. Furthermore, EoT + patients had higher levels of biomarkers indicative of sympathoadrenal activation (adrenaline and noradrenaline) and endothelial activation/dysfunction (sTM).

EoT + patients more frequently required in-hospital transfusion of blood products. There was also a trend towards fewer hospital-free days, but it was not significant $(p=0.06)$. The mortality rate was similar between groups (Table 2). The frequency of complications was higher in patients with EoT+. For example, all patients in our cohort that had acute kidney injury $(n=11 ; p<0.0001)$ and acute respiratory distress syndrome $(n=4 ; p=0.023)$ had EoT + . When accounting for the frequency of all complications associated with endotheliopathy (pulmonary embolism, deep vein thrombosis, sepsis, acute respiratory distress syndrome (ARDS) and acute kidney injury), the proportion of EoT+ patients with one or more complications was four times higher than that of the EoT- group ( $n=19,38.0 \%$ vs $9.2 \%, n=7 ; p=0.003)$.

\section{Syndecan-1 shedding in isolated TBI}

Because we wanted to determine how the breakdown of the glycocalyx contributed to poor outcome in the context of TBI, we performed a subgroup analysis including patients with isolated TBI only. Following a purposeful selection of variables approach to covariate selection, we fitted a multiple logistic regression to assess whether increasing shedding of syndecan-1 was associated with higher 72-h 


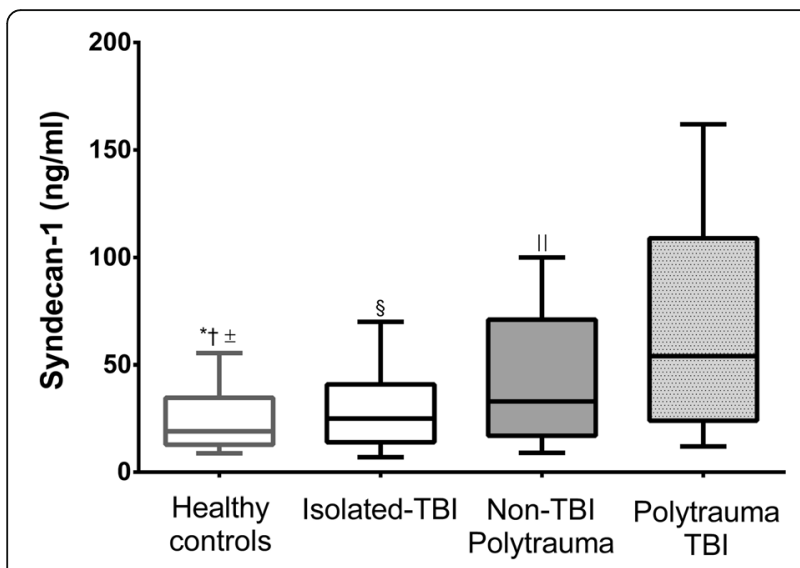

Fig. 2 Admission syndecan-1 levels in healthy volunteers $(n=29)$, patients with isolated TBI $(n=58)$, TBI combined with polytrauma $(n=68)$ and polytrauma only $(n=205)$. * Statistically significant difference between Isolated TBI patients vs. Healthy controls. † Statistically significant difference between Healthy controls vs. Non-TBI polytrauma patients. \pm Statistically significant difference between Healthy controls vs. Polytrauma TBI patients. § Statistically significant difference between Isolated TBI patients vs. Polytrauma TBI patients. || Statistically significant difference between Polytrauma TBI patients vs. Non-TBI polytrauma patients.

mortality. We chose deaths within $72 \mathrm{~h}$ of hospital arrival as the majority of deaths in our trauma center occur within this period [1]. We initially considered demographic data, injury severity indices, vital signs and syndecan-1 as variables that could be clinically meaningful, but only included in the preliminary effects model those variables with a $p$-value of less than 0.25 . The variables that met these criteria were age, arrival systolic blood pressure, syndecan-1, rTEG MA and ISS. After covariate selection and fitting the final effects model, we found that along with older age, higher ISS and lower rTEG MA, increasing syndecan-1 levels were independently associated with a higher likelihood of dying at 30 days (Table 3). We checked for potential interactions between syndecan-1 levels and ISS, and syndecan-1 levels and rTEG MA. None of the interactions were statistically significant or influenced importantly the effect of the other variable in the interaction term, thus they were not included in the final effects model. The Hosmer-Lemeshow test for goodness-of-fit of the model was not statistically significant, indicating a good fit of the model (goodness-of-fit test $p=0.8$ ). Based on this model, we evaluated the marginal effects at different levels of syndecan- 1 on the predicted probability of death at $72 \mathrm{~h}$ while all other covariates were left at their observed values. Our estimation suggests that, at least in this sample, the predicted probability of 72-h mortality rises with syndecan-1 levels in patients with isolated TBI and is about 26\% [95\% CI 17-35.2\%] for patients with syndecan-1 levels of $40 \mathrm{ng} / \mathrm{ml}$. For levels of equals of more than $100 \mathrm{ng} / \mathrm{ml}$ (13.8\% of patients had levels of or above this threshold), the predicted probability is at least $35.3 \%$ (Fig. 3).

\section{Discussion}

The current study provides evidence of the presence of EoT after TBI and supports the notion that breakdown of the glycocalyx is a contributor to the physiological derangements after TBI. In a cohort of severely injured patients, we found that in patients with isolated TBI, rising syndecan-1 levels were an independent factor associated with higher odds of 72 -h mortality. Furthermore, we found that polytrauma in the presence of TBI exacerbated the breakdown of the glycocalyx, leading to increased circulating levels of syndecan-1 and a higher frequency of EoT (defined as syndecan-1 $\geq 40 \mathrm{ng} / \mathrm{ml}$ ) in these patients compared to those with isolated TBI or polytrauma only.

Polytrauma in combination with TBI potentiated glycocalyx breakdown, resulting in a higher frequency of EoT + in these patients. This could have arisen from the activation of the sympathetic nervous system, which seems to drive endothelial dysfunction, glycocalyx breakdown, and coagulopathy both in severely injured trauma patients and in those with isolated TBI [22, 23]. Interestingly, sTM levels were comparable across the three groups (non-TBI polytrauma, polytrauma TBI and isolated TBI) indicating a similar degree of endothelial activation/dysfunction.

TBI patients with syndecan $-1 \geq 40 \mathrm{ng} / \mathrm{ml}(\mathrm{EoT}+)$ had physiological derangements shortly after injury and were more likely to have lower arrival systolic blood pressure, higher heart rate and base deficit, altered rTEG parameters compatible with coagulopathy (lower MA and prolonged ACT) and increased sympathoadrenal activation. Both hemodynamic instability and coagulopathy are components of EoT $[8,25]$. sTM is a marker of endothelial activation or dysfunction and it is expected to increase with higher glycocalyx breakdown (i.e. syndecan-1 shedding) $[8,25]$. The fact that TBI patients with EoT+ had significantly higher levels of adrenaline and sTM is consistent with the current notion of how endotheliopathy ensues, where the exacerbated sympathoadrenal activation following injury triggers glycocalyx breakdown, which leads to endothelial dysfunction and ultimately to endotheliopathy [8]. Our findings are consistent with what was reported by Di Battista and colleagues in a study conducted in patients with blunt isolated TBI. In this study, the authors found that in patients with blunt isolated TBI, the levels of biomarkers of sympathoadrenal activation (adrenaline and noradrenaline) co-varied with biomarkers of endotheliopathy including syndecan-1.

The breakdown of the glycocalyx exposes endothelial cells to circulating cytokines, immune cells, and other factors that trigger several responses, potentially leading 
Table 2 Clinical characteristics and outcomes comparing 126 TBI patients (isolated TBI and TBI and polytrauma) with EoT+ (syndecan-1 $\geq 40 \mathrm{ng} / \mathrm{ml}$ ) vs. EoT- (syndecan-1 $<40 \mathrm{ng} / \mathrm{ml}$ ) and 205 polytrauma patients without TBl

\begin{tabular}{|c|c|c|c|c|c|c|}
\hline & \multicolumn{2}{|l|}{ Polytrauma only } & \multirow[t]{2}{*}{$P$ value } & \multicolumn{2}{|l|}{$\underline{\mathrm{TB}}$} & \multirow[t]{2}{*}{$P$ value } \\
\hline & EOT- $(N=123)$ & EoT+ $(N=82)$ & & EoT- $(N=76)$ & EoT+ $(N=50)$ & \\
\hline ISS & 17 [13-26] & 24 [17-30] & 0.001 & 25 [18-29] & $32[26-41]$ & $<0.001$ \\
\hline $\mathrm{SBP}, \mathrm{mmHg}$ & 103 [88-126] & 120 [98-142] & 0.001 & 135 [116-150] & 115 [100-140] & 0.006 \\
\hline $\mathrm{HR}$, beats/minute & 96 [84-114] & $97[81-120]$ & 0.9 & 84 [70-104] & $100[84-111]$ & 0.007 \\
\hline $\mathrm{BE}, \mathrm{mEq} / \mathrm{l}$ & $-3[-6$ to 0$]$ & $-6[-8$ to -2$]$ & 0.002 & $-3[-5$ to 0$]$ & $-5[-8$ to -1$]$ & 0.01 \\
\hline GCS & 13 [3-15] & 7 [3-15] & 0.34 & 3 [3-9] & $3[3-7]$ & 0.7 \\
\hline Polytrauma, n (\%) & - & - & & $31(39.2)$ & 39 (70.9) & $<0.0001$ \\
\hline \multicolumn{7}{|l|}{ rTEG values } \\
\hline$A C T$, seconds & 113 [105-121] & 121 [113-125] & 0.04 & 105 [105-121] & 121 [105-136] & 0.004 \\
\hline $\mathrm{MA}, \mathrm{mm}$ & $65[62-69]$ & 63 [58-68] & 0.03 & 65 [60-69] & $62[58-67]$ & 0.006 \\
\hline a- angle, degrees & 75 [72-78] & 73 [70-77] & 0.004 & 73 [70-77] & 72 [69-76] & 0.12 \\
\hline \multicolumn{7}{|l|}{ Biomarkers } \\
\hline sTM, ng/ml & $5.0[3.8-6.9]$ & $7.9[5.6-9.4]$ & $<0.001$ & $5.1[4.3-7.2]$ & 7.4 [5.8-9.9] & $<0.0001$ \\
\hline Syndecan-1, ng/ml & 19.3 [11.3-30.2] & 94.4 [59.0-155.6] & N/A & 17.9 [11.6-30.5] & $109.6[71.1-217.8]$ & N/A \\
\hline Adrenaline $^{a}, \rho g / m l$ & 93.4 [36.7-287.6] & 185.7 [64.4-618.8] & 0.01 & 106.7 [38.0-311.4] & 339.0 [132.6-786.5] & $<0.001$ \\
\hline Noradrenaline $^{\mathrm{a}}, \mathrm{pg} / \mathrm{ml}$ & 846.2 [312.9-1340.3] & $1530.2[570.1-1825.9]$ & 0.03 & 588.9 [345.9-1148.9] & $1143.1[589.2-1866.1]$ & $<0.001$ \\
\hline \multicolumn{7}{|l|}{ Outcomes } \\
\hline Transfused, n (\%) & 49 (39.8) & $65(79.3)$ & $<0.01$ & $36(47.4)$ & $37(74.0)$ & 0.003 \\
\hline Hospital-free days & 18 [6-25] & $12[0-21]$ & 0.03 & $10[0-20]$ & $2[0-12]$ & 0.06 \\
\hline ICU-free days & 27 [20-30] & 23 [11-29] & 0.01 & $17[0-27]$ & $12[0-22]$ & 0.14 \\
\hline 24-h mortality, n (\%) & $1(0.8)$ & $6(7.3)$ & 0.02 & $5(6.6)$ & $5(10.0)$ & 0.5 \\
\hline 48-h mortality, n (\%) & $4(3.2)$ & $9(11.0)$ & 0.04 & $12(22.4)$ & $9(18.0)$ & 0.8 \\
\hline 72-h mortality, n (\%) & $6(4.9)$ & $10(12.2)$ & 0.07 & $12(22.4)$ & $12(24.0)$ & 0.25 \\
\hline 30-day mortality, n (\%) & $13(10.6)$ & $14(17.1)$ & 0.1 & $24(31.5)$ & $17(34.0)$ & 0.6 \\
\hline
\end{tabular}

Abbreviations: TBI traumatic brain injury, EOT traumatic endotheliopathy, ISS Injury Severity Score, AIS Abbreviated Injury Score, SBP systolic blood pressure, HR heart rate, $B E$ base excess, GCS Glasgow Coma Scale, rTEG rapid thrombelastography, $A C T$ activated clotting time, MA maximum amplitude, sTM soluble thrombomodulin, ICU intensive care unit

${ }^{\mathrm{a}}$ Data available for only 226 patients

Table 3 Univariate and multivariable logistic regression analyses of factors associated with 30-day in-hospital mortality in 58 patients with isolated traumatic brain injury

\begin{tabular}{|c|c|c|c|c|}
\hline & \multicolumn{2}{|l|}{ Univariate $(n=58)$} & \multicolumn{2}{|l|}{ Multivariate $(n=58)$} \\
\hline & $\overline{\beta(95 \% \mathrm{Cl})}$ & $\overline{p \text {-value }}$ & $\overline{\beta(95 \% \mathrm{Cl})}$ & $p$-value \\
\hline Syndecan-1 (10 ng/ml) & $0.04(-0.02-0.10)$ & 0.1 & $0.14(0.02-0.26)$ & 0.02 \\
\hline Age (yr) & $0.05(0.02-0.07)$ & 0.001 & $0.06(0.02-0.1)$ & 0.005 \\
\hline ISS & $0.2(0.09-0.32)$ & 0.001 & $0.29(0.1-0.46)$ & 0.001 \\
\hline rTEG MA (mm) & $-0.8(-0.14$ to -0.01$)$ & 0.02 & $-0.15(-0.2$ to -0.003$)$ & 0.04 \\
\hline Systolic blood pressure $(\mathrm{mmHg})$ & $-0.12(-0.3-0.01)$ & 0.18 & $-0.02(-0.05-0.01)$ & 0.3 \\
\hline \multicolumn{5}{|l|}{ Interactions } \\
\hline Syndecan-1 x ISS & NA & NA & $0.002(-0.48-0.08)$ & 0.72 \\
\hline Syndecan-1 x rTEG MA & NA & NA & $-0.01(-0.05-0.03)$ & 0.59 \\
\hline
\end{tabular}

Regression coefficients $(\beta)$ with $95 \%$ confidence intervals $(95 \% \mathrm{Cl})$ and $p$ values are displayed 


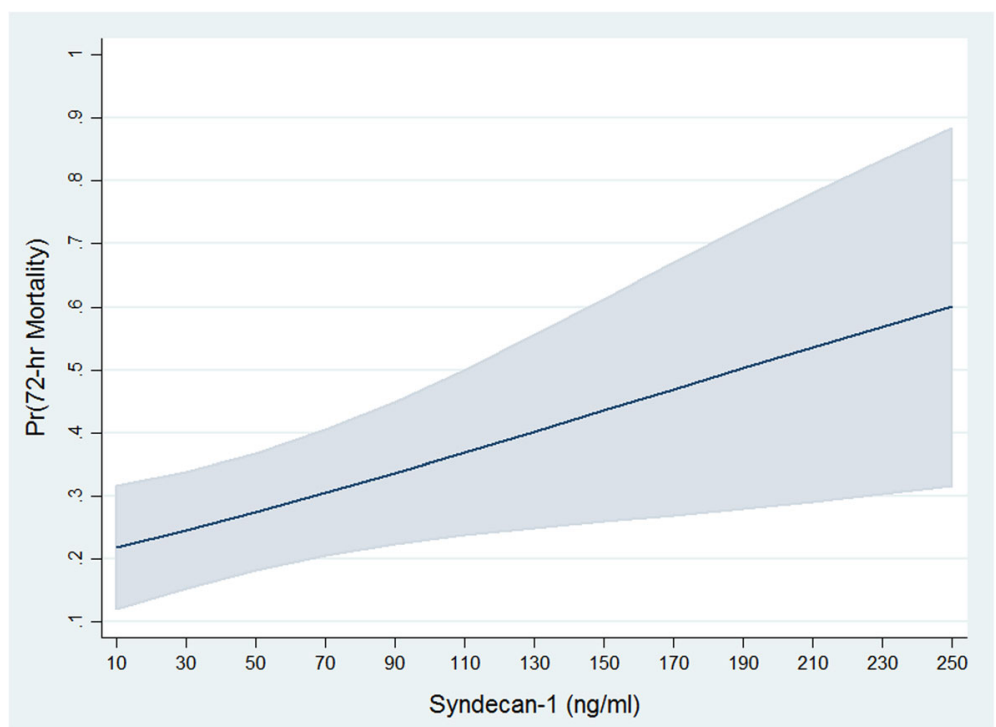

Fig. 3 Marginal plot of the effect of ED syndecan-1 level on the predicted probability of 72-h mortality ${ }^{*}$ in 58 patients with isolated TBI ${ }^{*}$ Shaded region indicates $95 \%$ confidence intervals

to EoT $[10,12,13,21,27]$. Until recently, most clinical studies have only evaluated the integrity of the endothelium and glycocalyx in the context of trauma-induced coagulopathy after TBI $[23,28]$. However, since the glycocalyx is involved in a series of physiological responses, its breakdown and associated EoT can have far greater implications $[6,18]$. For example, we can speculate that the glycocalyx plays a role in the regulation of the blood-brain barrier's permeability and that glycocalyx breakdown may contribute to the development of cerebral edema or to hemorrhagic progression. In a model of combined TBI and hemorrhagic shock, Halaweish et al. $[29,30]$ demonstrated that early treatment with fresh frozen plasma reduces the size of the brain lesion, attenuates endothelial activation, and improves neurological recovery. These positive effects of plasma could be to some extent attributable to the attenuation of the EoT through the repair of the glycocalyx $[18,31,32]$.

One of the main goals of this study was to determine whether the breakdown of the glycocalyx was associated with poor outcomes in patients with TBI. We found here that after adjusting for potential confounders, increasing syndecan-1 levels were an independent factor associated with higher odds of 72-h mortality in patients with isolated TBI. In addition, we did not find any significant interactions between syndecan-1 levels and ISS score, indicating that in these patients, the increased shedding of the glycocalyx is not merely a direct result of the severity of anatomical injury. These findings suggest that even though patients with isolated TBI seem to have less severe EoT, the breakdown of the glycocalyx may be significant enough to be a contributing factor to poor outcomes. When comparing TBI patients with and without EoT, we could only observe a trend towards fewer hospital-free days in patients with TBI and EoT+, but this trend was not statistically significant. In addition, we could not observe any differences in the frequency of mortality between these two groups. Patients in both groups were severely injured (ISS $>25$ ) irrespective of EoT status, which could have limited our capacity to observe small differences in these parameters in a relatively small cohort of patients. One of the components of the EoT is tissue injury and organ dysfunction [9]. Several studies have reported that sepsis, acute kidney injury and thrombosis are in part the result of endothelial dysfunction and glycocalyx breakdown [25]. The lung is one of the organs with the highest surface of glycocalyx. Pulmonary inflammation and vascular leakage mediated by glycocalyx breakdown are hallmarks of ARDS [33]. Although the frequency of ARDS is very low in our trauma center due to the limited use of crystalloids, all ARDS cases in this cohort had syndecan-1 levels $>40 \mathrm{ng} / \mathrm{ml}$. EoT+ patients with TBI were significantly more likely to have at least one of the aforementioned complications. The manifestation of these complications along with an exacerbated sympathoadrenal surge, increased glycocalyx breakdown, endothelial dysfunction and coagulopathy suggests that the traumatic endotheliopathy, as a systemic "disease", also occurs following TBI.

This study has several limitations. We included only patients with blunt trauma and moderate to severe ISS with radiologically confirmed TBI; however, by limiting our cohort to these patients, we were ensuring that we were studying patients who could benefit from potential 
early interventions that target the glycocalyx. Another limitation comes from employing head AIS and head CT scan to identify patients that had severe TBI. As opposed to head AIS, GCS scoring can be employed in the field to identify patients with altered mental status, and it is widely used to assess head injury severity. However, about half of the patients classified as Non-TBI polytrauma arrived with altered mental status (arrival GCS below thirteen) and had no confirmation of TBI. This is likely due to interventions on the field, for example, intubation, or other causes such as intoxication. Subsequently, we decided to use head AIS and CT confirmation to identify those patients that suffered from severe TBI. Mortality rates in TBI patients could be confounded by the withdrawal of life-sustaining therapies, which could predispose older patients to have life-sustaining therapies withdrawn at an earlier stage because of a perceived poor prognosis [34]. Turgeon and colleagues found that most deaths in TBI patients occurred after the withdrawal of life-sustaining therapies [35], a limitation shared by several studies with human TBI patients. Nonetheless, after controlling for age (and other factors), we found that increasing levels of syndecan-1 were an independent factor of mortality in patients with isolated TBI. We evaluated circulating syndecan- 1 as the defining biomarker of EoT, but several other unknown mechanisms are involved in this syndrome. Because the biomarker levels measured correspond to circulating levels that result from breakdown of the endothelial glycocalyx of blood vessels systemically, we cannot know how extensive the breakdown of the glycocalyx of cerebral blood vessels is. Nevertheless, previous studies from our group as well as substantial animal and clinical evidence support the role of circulating syndecan-1 in EoT and its associations with poor outcomes in trauma patients. The relatively small sample size in the isolated and polytrauma TBI groups is also a limitation. Nevertheless, we were able to detect statistically significant differences in levels of syndecan-1 across the groups $[8,12,23-25,27]$.

\section{Conclusions}

In conclusion, TBI combined with polytrauma markedly exacerbates glycocalyx breakdown, leading to a higher frequency of EoT+ than does isolated TBI or polytrauma without TBI. TBI patients with EoT+ (syndecan-1 $\geq 40 \mathrm{ng} / \mathrm{ml}$ ) exhibit marked endothelial dysfunction, physiological and coagulation derangements, and a higher frequency of complications. Even though patients with isolated TBI seem to experience less glycocalyx breakdown, as measured by circulating levels of syndecan-1, after adjusting for several factors including injury severity, increasing levels of this biomarker were an independent factor associated with higher 72-h mortality in this population.

\begin{abstract}
Abbreviations
AIS: Abbreviated Injury Scale; ARDS: Acute respiratory distress syndrome; CT: Computed tomography; ED: Emergency room; EOT: Traumatic endotheliopathy; GCS: Glasgow coma score; IQR: Interquartile range; ISS: Injury severity score; MIH: Multiple intracranial hemorrhage; rTEG: Rapid thrombelastography; sTM: Soluble thrombomodulin; TBI: Traumatic brain injury
\end{abstract}

\section{Acknowledgements}

We thank all our research assistants for their hard work and effort.

\section{Funding}

This study was supported by The Center for Translational Injury Research of The University of Texas Health Science Center and the William Stamp Farish Fund and the Howell Family Foundation.

\section{Availability of data and materials}

The datasets generated and analyzed during the current study are available in the Mendeley repository, [https://doi.org/10.17632/svt33j8cyz.1] [36]

\section{Authors' contributions}

EG-R contributed to conception of the study, data acquisition, analyses and interpretation of data, drafted the figures and wrote the article. JCC, CSC, RSK, $J \mathrm{~S}, \mathrm{JBH}, \mathrm{PIJ}$, contributed to acquisition of data and revised the article critically. CEW contributed to conception of the study, interpretation of data and revised the article critically. All authors read and approved the final article.

\section{Ethics approval and consent to participate}

Approval was obtained from The University of Texas Health Science Center at Houston Institutional Review Board (HSC-GEN-12-0059). Consent was obtained from the patient or a legally authorized representative within $72 \mathrm{~h}$ of admission or waived for patients who were discharged or died within $24 \mathrm{~h}$ of hospital admission.

Competing interests

The authors declare that they have no competing interests.

\section{Publisher's Note}

Springer Nature remains neutral with regard to jurisdictional claims in published maps and institutional affiliations.

\section{Author details}

${ }^{1}$ Center for Translational Injury Research (CeTIR), Department of Surgery, McGovern Medical School, University of Texas Health Science Center, 6431 Fannin, MSB 5.204, Houston, TX 77030, USA. ${ }^{2}$ Section for Transfusion Medicine, Capital Region Blood Bank, Copenhagen University Hospital, Rigshospitalet, Blegdamsvej 9, DK-2100 Copenhagen, Denmark. ${ }^{3}$ Department of Anesthesia, Centre of Head and Orthopedics, Copenhagen University Hospital, Rigshospitalet, Copenhagen, Denmark. ${ }^{4}$ Department of Pediatric Surgery, McGovern Medical School at The University of Texas Health Science Center, 6431 Fannin, MSB 5.258, Houston, TX 77030, USA. ${ }^{5}$ Department of Neurosurgery, Mischer Neuroscience Institute, McGovern Medical School at The University of Texas Health Science Center, 6400 Fannin, Suite 2800, Houston, TX 77030, USA.

Received: 12 July 2018 Accepted: 2 November 2018

Published online: 21 November 2018

\section{References}

1. Oyeniyi BT, Fox EE, Scerbo M, Tomasek JS, Wade CE, Holcomb JB. Trends in 1029 trauma deaths at a level 1 trauma center: impact of a bleeding control bundle of care. Injury. 2017:48(1):5-12

2. Fox EE, Holcomb JB, Wade CE, Bulger EM, Tilley BC, Group PS. Earlier endpoints are required for hemorrhagic shock trials among severely injured patients. Shock. 2017;47(5):567-73. 
3. Wang KW, Chen HJ, Lu K, Liliang PC, Liang CL, Tsai YD, et al. Simvastatin attenuates the cerebral vascular endothelial inflammatory response in a rat traumatic brain injury. Ann Clin Lab Sci. 2014;44(2):145-50.

4. Holcomb JB, del Junco DJ, Fox EE, Wade CE, Cohen MJ, Schreiber MA, et al. The prospective, observational, multicenter, major trauma transfusion (PROMMTT) study: comparative effectiveness of a time-varying treatment with competing risks. JAMA Surg. 2013;148(2):127-36

5. Holcomb JB, Tilley BC, Baraniuk S, Fox EE, Wade CE, Podbielski JM, et al. Transfusion of plasma, platelets, and red blood cells in a 1:1:1 vs a 1:1:2 ratio and mortality in patients with severe trauma: the PROPPR randomized clinical trial. JAMA. 2015;313(5):471-82.

6. Chignalia AZ, Yetimakman F, Christiaans SC, Unal S, Bayrakci B, Wagener BM, et al. The glycocalyx and trauma: a review. Shock. 2016;45(4):338-48.

7. Alphonsus CS, Rodseth RN. The endothelial glycocalyx: a review of the vascular barrier. Anaesthesia. 2014;69(7):777-84.

8. Johansson PI, Henriksen HH, Stensballe J, Gybel-Brask M, Cardenas JC, Baer LA, et al. Traumatic endotheliopathy: a prospective observational study of 424 severely injured patients. Ann Surg. 2017;265(3):597-603.

9. Holcomb JB, Pati S. Optimal trauma resuscitation with plasma as the primary resuscitative fluid: the surgeon's perspective. Hematology Am Soc Hematol Educ Program. 2013;2013:656-9.

10. Johansson P, Stensballe J, Ostrowski S. Shock induced endotheliopathy (SHINE) in acute critical illness - a unifying pathophysiologic mechanism. Crit Care. 2017;21(1):25

11. Rahbar E, Baer LA, Cotton BA, Holcomb JB, Wade CE. Plasma colloid osmotic pressure is an early indicator of injury and hemorrhagic shock. Shock. 2014; 41(3):181-7.

12. Rahbar E, Cardenas JC, Baimukanova G, Usadi B, Bruhn R, Pati S, et al. Endothelial glycocalyx shedding and vascular permeability in severely injured trauma patients. J Transl Med. 2015;13:117.

13. Torres Filho I, Torres LN, Sondeen JL, Polykratis IA, Dubick MA. In vivo evaluation of venular glycocalyx during hemorrhagic shock in rats using intravital microscopy. Microvasc Res. 2013;85:128-33.

14. Torres Filho IP, Torres LN, Salgado C, Dubick MA. Plasma syndecan-1 and heparan sulfate correlate with microvascular glycocalyx degradation in hemorrhaged rats after different resuscitation fluids. Am J Physiol Heart Circ Physiol. 2016;310(11):H1468-78.

15. Gonzalez Rodriguez E, Cardenas JC, Lopez E, Cotton BA, Tomasek JS, Ostrowski SR, et al. Early identification of the patient with endotheliopathy of trauma by arrival serum albumin. Shock. 2018:50(1):31-37.

16. Ban K, Peng Z, Pati S, Witkov RB, Park PW, Kozar RA. Plasma-mediated gut protection after hemorrhagic shock is lessened in syndecan-1-/- mice. Shock. 2015;44(5):452-7.

17. Haywood-Watson RJ, Holcomb JB, Gonzalez EA, Peng Z, Pati S, Park PW, et al. Modulation of syndecan-1 shedding after hemorrhagic shock and resuscitation. PLoS One. 2011;6(8):e23530.

18. Kozar RA, Pati S. Syndecan-1 restitution by plasma after hemorrhagic shock. J Trauma Acute Care Surg. 2015;78(6 Suppl 1):S83-6.

19. Kozar RA, Peng Z, Zhang R, Holcomb JB, Pati S, Park P, et al. Plasma restoration of endothelial glycocalyx in a rodent model of hemorrhagic shock. Anesth Analg. 2011:112(6):1289-95.

20. Peng Z, Pati S, Potter D, Brown R, Holcomb JB, Grill R, et al. Fresh frozen plasma lessens pulmonary endothelial inflammation and hyperpermeability after hemorrhagic shock and is associated with loss of syndecan 1. Shock. 2013;40(3):195-202.

21. Sillesen M, Rasmussen LS, Jin G, Jepsen CH, Imam A, Hwabejire JO, et al. Assessment of coagulopathy, endothelial injury, and inflammation after traumatic brain injury and hemorrhage in a porcine model. J Trauma Acute Care Surg. 2014;76(1):12-9 discussion 9-20.

22. Di Battista AP, Rhind SG, Hutchison MG, Hassan S, Shiu MY, Inaba K, et al. Inflammatory cytokine and chemokine profiles are associated with patient outcome and the hyperadrenergic state following acute brain injury. J Neuroinflammation. 2016;13:40

23. Di Battista AP, Rizoli SB, Lejnieks B, Min A, Shiu MY, Peng HT, et al. Sympathoadrenal activation is associated with acute traumatic coagulopathy and endotheliopathy in isolated brain injury. Shock. 2016;46(3 Suppl 1):96-103

24. Gonzalez Rodriguez E, Ostrowski SR, Cardenas JC, Baer LA, Tomasek JS, Henriksen $\mathrm{HH}$, et al. Syndecan-1: a quantitative marker for the endotheliopathy of trauma. J Am Coll Surg. 2017;225:419.
25. Ostrowski SR, Henriksen HH, Stensballe J, Gybel-Brask M, Cardenas JC, Baer LA, et al. Sympathoadrenal activation and endotheliopathy are drivers of hypocoagulability and hyperfibrinolysis in trauma: a prospective observational study of 404 severely injured patients. J Trauma Acute Care Surg. 2017;82(2):293-301.

26. Cotton BA, Faz G, Hatch QM, Radwan ZA, Podbielski J, Wade C, et al. Rapid thrombelastography delivers real-time results that predict transfusion within 1 hour of admission. J Trauma. 2011;71(2):407-14 discussion 14-7.

27. Johansson PI, Stensballe J, Rasmussen LS, Ostrowski SR. A high admission syndecan-1 level, a marker of endothelial glycocalyx degradation, is associated with inflammation, protein C depletion, fibrinolysis, and increased mortality in trauma patients. Ann Surg. 2011; 254(2):194-200.

28. Genet GF, Johansson PI, Meyer MA, Solbeck S, Sorensen AM, Larsen CF, et al. Trauma-induced coagulopathy: standard coagulation tests, biomarkers of coagulopathy, and endothelial damage in patients with traumatic brain injury. J Neurotrauma. 2013;30(4):301-6.

29. Halaweish I, Bambakidis T, Nikolian VC, Georgoff P, Bruhn P, Piascik P, et al. Early resuscitation with lyophilized plasma provides equal neuroprotection compared with fresh frozen plasma in a large animal survival model of traumatic brain injury and hemorrhagic shock. J Trauma Acute Care Surg. 2016:81(6):1080-7.

30. Halaweish I, Bambakidis T, He W, Linzel D, Chang Z, Srinivasan A, et al. Early resuscitation with fresh frozen plasma for traumatic brain injury combined with hemorrhagic shock improves neurologic recovery. J Am Coll Surg. 2015:220(5):809-19.

31. Wu F, Peng Z, Park PW, Kozar RA. Loss of syndecan-1 abrogates the pulmonary protective phenotype induced by plasma after hemorrhagic shock. Shock. 2017;48:340,

32. Pati S, Potter DR, Baimukanova G, Farrel DH, Holcomb JB, Schreiber MA. Modulating the endotheliopathy of trauma: factor concentrate versus fresh frozen plasma. J Trauma Acute Care Surg. 2016;80(4):576-84 discussion 84-5.

33. Brettner F, von Dossow V, Chappell D. The endothelial glycocalyx and perioperative lung injury. Curr Opin Anaesthesiol. 2017:30(1):36-41.

34. McCredie VA, Alali AS, Xiong W, Rubenfeld GD, Cuthbertson BH, Scales DC, et al. Timing of withdrawal of life-sustaining therapies in severe traumatic brain injury: impact on overall mortality. J Trauma Acute Care Surg. 2016; 80(3):484-91.

35. Turgeon AF, Lauzier F, Simard J-F, Scales DC, Burns KEA, Moore L, et al. Withdrawing life-sustaining therapy for patients with severe traumatic brain injury. CMAJ. 2011;183(14):1570-1.

36. Gonzalez Rodriguez E, Cardenas JC, Cox CS, Kitagawa RS, Stensballe J, Holcomb JB, et al. Traumatic brain injury is associated with increased syndecan-1 shedding in severely injured patients dataset. $\mathrm{V} 1 \mathrm{ed}$. Mendeley 2018.

Ready to submit your research? Choose BMC and benefit from

- fast, convenient online submission

- thorough peer review by experienced researchers in your field

- rapid publication on acceptance

- support for research data, including large and complex data types

- gold Open Access which fosters wider collaboration and increased citations

- maximum visibility for your research: over $100 \mathrm{M}$ website views per year

At $\mathrm{BMC}$, research is always in progress.

Learn more biomedcentral.com/submission 